\title{
Exposição a Fatores de Risco de Adolescentes em Acolhimento Institucional no Sul do Brasil
}

\author{
Josiane Lieberknecht Wathier Abaid" \\ Universidade Federal do Rio Grande do Sul, Porto Alegre, Brasil \\ Centro Universitário Franciscano, Santa Maria, Brasil \\ Débora Dalbosco Dell'Aglio \\ Universidade Federal do Rio Grande do Sul, Porto Alegre, Brasil
}

\begin{abstract}
RESUMO
Este estudo buscou identificar a exposição a fatores de risco em adolescentes que vivem em instituições de acolhimento, em duas cidades do sul do Brasil. Participaram 113 adolescentes de 12 a 18 anos $(M=14,82 ; d p=1,47)$, sendo $60,2 \%$ meninas, que responderam individualmente a um questionário para investigar fatores de risco e proteção. Foi observada uma permanência média de mais de seis anos nas instituições de acolhimento, embora com manutenção do contato familiar para mais de $70 \%$ dos participantes. Foram identificados diversos fatores de risco ao desenvolvimento, como repetência escolar e exposição à violência intra e extrafamiliar, entre outros. Evidencia-se o desafio à instituição que acolhe e a necessidade de investimento em políticas públicas que auxiliem na recuperação e fortalecimento dos laços familiares.
\end{abstract}

Palavras-chave: acolhimento institucional; adolescentes; fatores de risco.

\section{ABSTRACT \\ Exposure to Risk Factors of Adolescents in Residential Care in the South of Brazil}

This research aimed to identify the exposition to risk factors in adolescents from the South of Brazil who lived in residential care. Participants were 113 adolescents aged between 12 and 18 years old $(\mathrm{M}=14.82$; sd=1.47), $60.2 \%$ girls, who answered to the questionnaire applied individually to investigate risk and protective factors. The adolescents remained on the residential care more than six years, however, their family contact was preserved, regarding more than $70 \%$ of the participants. The results showed different risk factors to development, such as school failure and violence exposition in family and community, among others. The challenge of residential care and the need for investment in public policies that may assist in the recovery and strengthening of family ties are evident.

Keywords: residencial care; adolescents; risk factors.

Morar temporariamente longe de suas famílias de origem é a realidade de milhares de crianças e adolescentes no Brasil, que foram acolhidos institucionalmente. $\mathrm{O}$ acolhimento institucional é uma medida protetiva prevista no Estatuto da Criança e do Adolescente (ECA) que visa garantir um contexto de desenvolvimento adequado até que seja possível o retorno à família de origem ou a colocação em família substituta (Brasil, 1990).

Na perspectiva legislativa atual, a criança e o adolescente são vistos como sujeitos de direitos, e ficam em condições diferenciadas dependendo da necessi- dade de proteção ou socioeducação. Na mesma direção, as recomendações da Lei 8.069/90, da instituição do ECA, foram aperfeiçoadas na Lei 12.010, sancionada em agosto de 2009, que altera a sistemática prevista para garantia do direito à convivência familiar a todas as crianças e adolescentes.

Segundo Guará (2006), frente às determinações do ECA (Brasil, 1990), a primeira providência desempenhada pelas instituições foi reordenar a sua estrutura física, transformando grandes espaços em pequenas salas e quartos, ou mesmo, diminuindo o número de acolhidos. Por outro lado, as determinações propostas

* Endereço para correspondência: Josiane Lieberknecht Wathier Abaid - josianelieb@yahoo.com.br 
pelo novo estatuto não foram atreladas apenas às condições físicas dos locais, mas também à ideia da implementação de um novo programa socioeducativo a ser desenvolvido pela instituição. Do contrário, pequenas residências reproduziriam o mesmo sistema de atendimento das grandes instituições (Guará, 2006), trazendo repercussões negativas ao desenvolvimento das crianças e adolescentes acolhidos institucionalmente.

Estudos apontam tanto aspectos de risco como de proteção ao desenvolvimento relacionados à institucionalização. Sabe-se que a colocação num contexto não familiar e a permanência longe da família de origem podem atuar como mais uma violência para crianças e adolescentes que já passaram por situações de maus-tratos ao longo de seu desenvolvimento. A questão do abandono e a situação de acolhimento institucional interferem na vinculação afetiva de modo significativo em crianças em instituições, à espera de adoção (Oliveira \& Próchno, 2010). Adolescentes que passaram parte do seu desenvolvimento em instituições podem ter, ainda, o seu pensamento comprometido, bem como o desenvolvimento da linguagem, o motor e o funcionamento intelectual (Velarde \& Martínez, 2008). Na mesma direção, Sigal, Perry, Rossignol e Ouimet (2003) afirmam que a experiência de institucionalização prolongada e precoce pode trazer prejuízos graves ao desenvolvimento cognitivo dos indivíduos. Além disso, o tempo de institucionalização pode influenciar a rede de apoio, especialmente quanto à percepção de proximidade e qualidade das relações, visto que maior tempo de institucionalização pode associar-se a pior percepção de proximidade nas relações e menor número de contatos na rede de apoio (Siqueira \& Dell'Aglio, 2010). Aspectos como alta rotatividade de funcionários, falta de atividades planejadas e de apoio afetivo, que interferem diretamente no apoio social, podem trazer prejuízos aos jovens que se desenvolvem em instituições (Azor \& Vectore, 2008; Carvalho, 2002; Ribeiro \& Ciampone, 2002), sobretudo aqueles com menor frequência de convivência familiar (Prada \& Weber, 2002; Prada, Williams, \& Weber, 2007).

Entretanto, apesar da complexidade de fatores de risco potencialmente envolvidos na institucionalização, há estudos demonstrando mudanças significativas no ambiente e na qualidade de atendimento destes locais (Arpini \& Lopes, 2009; Cavalcante, Magalhães, \& Pontes, 2007; Siqueira \& Dell'Aglio, 2006; Siqueira \& Dell'Aglio, 2010), sendo que as instituições de proteção têm se mostrado mais adequadas ao atendimento e proteção de crianças e adolescentes, de acordo com as especificações do ECA (Brasil, 1990). Na perspectiva bioecológica, o desenvolvimento humano constitui-se de forma mais ampla do que as condições contextuais em que este ocorre (Bronfenbrenner, 1979/1996). Por exemplo, um importante aspecto promotor do desenvolvimento emocional é a relação com alguém que se importe genuinamente pela criança ou adolescente e estabeleça uma relação proximal com ela (Bronfenbrenner, 2011). Diversos estudos que consideram a perspectiva contextual do desenvolvimento têm demonstrado que ser acolhido temporariamente em casas-lares e abrigos residenciais pode contribuir para que o adolescente constitua uma rede de apoio satisfatória, capaz de propiciar sentimento de confiança e processos proximais que favorecem o seu desenvolvimento psicológico. Para Albornoz (2009), a institucionalização pode funcionar como um recurso terapêutico para a recuperação de crianças e adolescentes vítimas de abandono e abuso, uma vez que, nesse contexto, podem ser resgatadas as experiências de cuidado afetivo, não permitindo que as vivências de privação anteriores sejam incapacitantes. Após um período de adaptação inicial, Carvalho e Manita (2010) verificaram uma percepção positiva das crianças acolhidas em relação à vida na instituição e, mesmo, uma valorização positiva da instituição enquanto lar que os acolhe. Estudos têm apresentado resultados indicativos de presença de processos de resiliência na construção de novas relações afetivas estabelecidas após a institucionalização, tanto com pares, sobretudo irmãos (Alexandre \& Vieira, 2004), como com adultos (Dalbem \& Dell'Aglio, 2008) entre adolescentes em situação de acolhimento institucional. Além disso, existe a possibilidade de que o adolescente institucionalizado viva em um ambiente com rotinas e regras bem estabelecidas, capazes de auxiliar na ampliação do seu repertório comportamental e cognitivo, o que facilita seu desempenho escolar e social (Weber, 2007). Depreende-se, assim, que não há consenso quanto às repercussões da institucionalização e, devido a isso, são necessários mais estudos para investigar estes processos no desenvolvimento de crianças e adolescentes em acolhimento.

Atualmente, os dados oficiais publicados mais recentemente sobre esse contexto no país são do Levantamento Nacional de Abrigos Brasileiros para Crianças e Adolescentes, realizado pelo Instituto de Pesqui- 
sa Econômica Aplicada (IPEA) em 2003, a pedido da Subsecretaria de Promoção dos Direitos da Criança e do Adolescente e do Conselho Nacional dos Direitos da Criança e do Adolescente (CONANDA). Essa pesquisa investigou 589 instituições de acolhimento institucional que recebiam recursos do Governo Federal, onde viviam cerca de 20 mil crianças e adolescentes, sendo na maioria meninos entre 7 e 15 de idade, negros e pobres (Silva, 2004). O principal motivo do acolhimento institucional da população infanto-juvenil pesquisada foi a carência de recursos materiais da família $(24,1 \%)$, seguido do abandono pelos pais ou responsáveis $(18,8 \%)$ e da violência doméstica $(11,6 \%)$. Nesta mesma análise, $87 \%$ destas crianças e adolescentes abrigados tinham família, sendo que $58 \%$ deles mantinham vínculo com seus familiares e uma minoria $(5,8 \%)$ estava impedida judicialmente de manter contato com os familiares. Para Silva (2004), a precária condição socioeconômica das famílias parecia contribuir para a deterioração das relações familiares, e aumentaria a chance de períodos de institucionalização das crianças e adolescentes oriundas desses núcleos. Na região metropolitana de Campinas (SP), um estudo observou uma predominância de meninas com idade acima de 16 anos em acolhimentos institucionais (Coelho, Hirata, Barbette, Vieira, \& Oliveira, 2009). Quanto ao tempo de permanência nas instituições da pesquisa do IPEA, que variou de menos de um mês a mais de dez anos, mais da metade vivia há mais de dois anos nesse contexto e $32,9 \%$ dos institucionalizados estava neste contexto desde os dois aos cinco anos de idade (Silva, 2004). O levantamento apontou que na região sul havia $15,5 \%$ das crianças e adolescentes da pesquisa do IPEA (Silva, 2004), cujo motivo do ingresso nas instituições foi predominantemente devido a abandono $(21,6 \%)$ ou violência por parte de pais ou responsáveis $(15,5 \%)$. Apesar da determinação do ECA de que as instituições devem comunicar à Justiça sobre crianças e adolescentes acolhidos, ainda era baixo o percentual de processos nas varas de infância e juventude no Brasil (54,6\%). A região sul, apresentava um dos maiores percentuais de processos judiciais $(69,7 \%)$.

Em outro levantamento realizado pela Promotoria da Infância e Juventude do Estado do Rio Grande do Sul, entre os anos de 2006 e 2007, foi identificada a existência de 1.204 crianças e adolescentes acolhidos em instituições, distribuídas em 67 abrigos na região de Porto Alegre e região metropolitana (Gasperin, Luz, Souza, Perin, \& Tejadas, 2007). Em contraste parcial com os dados brasileiros obtidos em 2003, a maioria era de meninos, em situação de vulnerabilidade social, porém, brancos. Além disso, menos de $20 \%$ destas crianças e adolescentes recebiam visita da família. Em Seminário promovido pela Fundação de Proteção Especial do Rio Grande do Sul, em 2011, foi informado que $44 \%$ das crianças e adolescentes institucionalizadas ingressaram no sistema devido à violência física ou psicológica e $31 \%$ por pobreza e abandono de suas famílias (FPERGS, 2012). Dessa forma, observa-se que estes jovens estiveram expostos a diversos fatores de risco ao longo do seu desenvolvimento, os quais estão relacionados a variáveis ambientais ou contextuais que aumentam a probabilidade da ocorrência de algum efeito indesejável no desenvolvimento mental (Velarde \& Martínez, 2008). Considerando-se a necessidade de conhecer de forma mais ampla a questão da institucionalização na adolescência, o presente estudo buscou identificar a exposição a fatores de risco em adolescentes que vivem em instituições de proteção em Porto Alegre e Santa Maria, RS.

\section{MÉTODO}

A partir de um delineamento transversal e quantitativo, esta investigação ocorreu no contexto das instituições de acolhimento de gestão pública (estadual e municipal) e privada (ONGs que tinham convênio com as públicas). Na capital do Rio Grande do Sul, o governo estadual atualmente gerencia sete Núcleos de Abrigos Residenciais (NARES), divididos pelas regiões de Porto Alegre, sendo que cada núcleo é composto de quatro a dez abrigos residenciais. As crianças e os adolescentes recebidos são encaminhados para unidades residenciais de pequeno porte, com no máximo 15 acolhidos por residência, sob os cuidados de dois monitores fixos, que se alternam entre plantões. Estes abrigos residenciais estão inseridos na comunidade, na qual estas crianças e adolescentes utilizam recursos, tais como escola, centros de lazer, praças e também a rede municipal de saúde (Albornoz, 2009).

Por sua vez, as instituições municipais da capital do Estado do RS, de responsabilidade da Fundação de Assistência Social e Cidadania (FASC), atualmente oferecem dez Abrigos Residenciais Municipais (ARMs) da rede própria, que atendem até 20 crianças e adolescentes e possuem equipe técnica (assistente social, psicólogo, pedagogo, etc) e educadores sociais. Além disso, existem 44 casas lares, administradas por ONGs e conveniadas com o município. Cada Casa Lar 
deve ter no máximo oito jovens, além da cuidadora e também poderá contar com o cônjuge da cuidadora residente (chamado anteriormente de "pai social").

Na cidade de Santa Maria há quatro instituições, sendo duas do município, uma com atendimento a meninas adolescentes e outra a meninos, mas administradas de forma terceirizada por uma ONG. As demais são ONGS com diferentes perfis. Uma delas funciona na modalidade de casas lares, com uma cuidadora residente.

\section{Participantes}

Participaram 113 adolescentes de 12 a 18 anos $(M=14,82 ; d p=1,47)$, sendo $60,2 \%$ meninas, que estavam em instituições de acolhimento de gestão pública (estadual e municipal) e privada (organizações não governamentais que tinham convênio com as públicas), nas cidades de Porto Alegre $(79,8 \%$ dos participantes) e Santa Maria (20,2\% dos participantes), Rio Grande do Sul. Na ocasião da coleta de dados, a cidade de Santa Maria era a única do interior do estado onde tinha outra unidade de uma mesma ONG em POA. Os critérios de inclusão foram: ter entre 12 e 18 anos, desejar colaborar com a pesquisa, estar há pelo menos 30 dias em acolhimento institucional e ter capacidade de compreender o questionário, conforme avaliação subjetiva da equipe técnica e da equipe de pesquisa durante a coleta de dados.

\section{Instrumentos}

Foi utilizada uma Ficha de dados sociodemográficos dos participantes (nome, idade, sexo, escolaridade, história de acolhimento, convivência familiar e contatos familiares) e o Questionário da Juventude Brasileira-Versão Fase II (QJB-II, Dell'Aglio, Koller, Cerqueira-Santos, \& Colaço, 2011), composto por 77 questões, sendo algumas de múltipla escolha e outras em formato Likert de cinco pontos sobre intensidade e frequência. Para este estudo foram utilizados os itens que avaliavam as seguintes variáveis: escolaridade, comportamento sexual, eventos estressores, violência intra e extrafamiliar, ideação e tentativas de suicídio. Foi avaliada a ocorrência de vinte eventos estressores, tais como "alguém em minha casa está desempregado", "já morei na rua", "alguém da minha família está ou esteve preso" e "alguém muito importante pra mim faleceu". Os itens foram dispostos no formato dicotômico ( $0=$ Não, $1=$ Sim) e o escore total foi computado somando-se o número de respostas positivas. Nos itens marcados como sim, questionou-se ainda o quanto o adolescente considerou estressante, com uma escala Likert de cinco pontos ( $1=$ Nada ruim, $5=$ Horrível $)$, de forma a identificar o impacto dos eventos.

\section{Procedimentos e considerações éticas}

O projeto de pesquisa foi aprovado pelo Comitê de Ética em Pesquisa do Instituto de Psicologia da UFRGS, sob o registro número 2009060. Foi solicitado o Termo de Concordância das Instituições governamentais e não governamentais, que mantêm a guarda dos adolescentes, autorizando a realização do estudo.

Os adolescentes que atendiam aos critérios de inclusão foram convidados a participar, tendo sido esclarecida a voluntariedade em responder aos instrumentos, a garantia de sigilo das informações pessoais e a possibilidade de desistência a qualquer momento, sem qualquer prejuízo. Os adolescentes que concordaram em participar da pesquisa também assinaram o Termo de Assentimento. O questionário foi aplicado nas dependências das instituições, de forma individual ou em pequenos grupos e com auxílio de um pesquisador previamente treinado para esse fim (heteroaplicação).

Os dados coletados foram analisados nesse estudo de forma descritiva (medidas de frequência e percentual para variáveis categóricas e de média e desviopadrão, quando numéricas) e inferencial (testes quiquadrado, correlação de Spearman) para identificar o perfil dos adolescentes, utilizando-se o software SPSS.

\section{RESULTADOS}

Dados relacionados ao acolhimento e características demográficas

Os adolescentes desse estudo estavam em acolhimento por um período entre um mês até 215 meses $(M=76,91 ; d p=55,29)$, o que representa mais de seis anos em média, e foram acolhidos entre uma e quatro vezes $(M=1,83 ; d p=0,88)$ durante esse tempo. A idade de entrada no primeiro acolhimento variou de zero a 16 anos $(\mathrm{M}=8,06 ; \mathrm{dp}=4,63)$. O perfil demográfico predominante foi o de meninas $(60,2 \%)$, não tendo sido encontrada diferença por sexo quanto ao tempo de institucionalização ou o número de vezes em que foram acolhidos. Quanto a doenças crônicas, 11,7\% afirmaram ter alguma, como asma, hepatite B e HIV e 26,55\% referiram estar em acompanhamento psicológico e/ou psiquiátrico. 
Apenas 6\% dos adolescentes trabalhavam de forma remunerada, exercendo atividades de aprendiz, tais como cuidar de crianças, empacotar em mercados, atividades administrativas, ente outros. Conforme a Tabela 1, a cor da pele predominante foi negra ou parda, sobre a qual também não houve diferença por sexo. Os pais dos adolescentes em geral não estavam mais juntos, sendo que em cerca de $30 \%$ dos casos algum dos pais já era falecido. Quinze participantes declararam ter ambos os pais falecidos. A média de irmãos consanguíneos, que variou de zero a 19 , é de $4,88(\mathrm{dp}=3,06)$, salientando-se que $21 \%$ dos adolescentes tinham pelo menos um irmão na mesma instituição (Tabela1).

\begin{tabular}{|c|c|c|c|}
\hline \multicolumn{4}{|c|}{ aticas } \\
\hline \multicolumn{2}{|l|}{ Característica } & $f$ & $\%$ \\
\hline \multicolumn{4}{|l|}{ Cor } \\
\hline \multicolumn{2}{|l|}{ Negra } & 45 & 39,8 \\
\hline \multicolumn{2}{|l|}{ Parda } & 33 & 29,2 \\
\hline \multicolumn{2}{|l|}{ Branca } & 31 & 27,4 \\
\hline \multicolumn{2}{|l|}{ Outras } & 4 & 3,6 \\
\hline \multicolumn{2}{|c|}{ Recebem benefício "Bolsa família" } & 26 & 59,1 \\
\hline \multicolumn{2}{|c|}{ Têm irmãos na mesma instituição } & 51 & 45,1 \\
\hline \multicolumn{2}{|c|}{ Têm irmãos em outra instituição } & 16 & 14,1 \\
\hline \multicolumn{2}{|c|}{ Têm irmãos com familiares } & 63 & 56,8 \\
\hline \multicolumn{2}{|c|}{ Têm irmãos com pessoas sem ligação familiar } & 26 & 23,6 \\
\hline \multicolumn{2}{|l|}{ Pais vivem juntos } & 14 & 14,6 \\
\hline \multirow[t]{2}{*}{ Conhece(eu) os pais } & Mãe & 80 & 71,4 \\
\hline & Pai & 62 & 54,9 \\
\hline \multirow[t]{2}{*}{ Pais falecidos } & Mãe & 30 & 26,5 \\
\hline & Pai & 37 & 32,7 \\
\hline
\end{tabular}

\section{Contato familiar}

A maior parte dos adolescentes do estudo $(77,7 \%)$ indicou manter contato com familiares consanguíneos e não houve diferença entre meninos e meninas quanto a isso. Para $19,44 \%$ dos adolescentes a pessoa considerada fonte de apoio mais importante no último ano foi um familiar, embora, para a maioria $(56,94 \%)$ tenha sido um funcionário (educador ou técnico) da própria instituição.

A maioria dos adolescentes indicou receber visita mensal $(37,2 \%)$, seguida da quinzenal $(22,1 \%)$ e também das visitas semanais (10,5\%). Quanto ao tempo de visitação, foi observado que a maior parte dos adolescentes participantes do estudo $(55,9 \%)$ informou que passava de 1 a 2 dias com suas famílias. Quanto mais idade tinha o adolescente ao ser acolhido pela primeira vez, maior foi a frequência de contato com família de origem durante a sua permanência na instituição $(\rho=0,236 ; p=0,03)$. Por outro lado, quanto maior o tempo de institucionalização, menor foi a frequência desse contato $(\rho=-0,26 ; p<0,02)$. Não houve diferença por sexo ou por tipo de instituição quanto à frequência do contato com os familiares.

\section{Aspectos relacionados à escola}

Em relação à escolaridade, os participantes cursavam de primeira série do Ensino Fundamental (3,6\%) até a segunda série do Ensino Médio (0,9\%), sendo que a maioria frequentava a $5^{\mathrm{a}}$ e $6^{\mathrm{a}}$ séries do Ensino Fundamental $(36,4 \%)$ em ensino público. Estudavam de oitava série a segundo ano do ensino médio 13 adolescentes. Apenas um estudava em escola particular e dois estavam sem escola no momento da coleta de dados (devido à transferência). Cinco deles estavam na Educação para Jovens e Adultos (EJA). A defasagem escolar reflete-se nos 82,3\% dos adolescentes que já reprovaram de ano, e a média de vezes em que isso ocorreu foi de 2,08 $(\mathrm{dp}=1,19)$. Alguns deles também declararam terem sido expulsos da escola $(14,2 \%)$. Destaca-se que muitos adolescentes desconhecem a escolaridade de seus pais $(63,7 \%$ dos participantes não sabem a escolaridade do pai e $45,7 \%$, da mãe). Entre os que indicaram o grau de estudo de seus pais, verificou-se que a mãe normalmente tinha menos anos de estudo do que o pai, sendo que a maior parte era analfabeta ou tinha escolaridade fundamental incompleta. 


\section{Eventos estressores e violência}

Foi realizado o levantamento dos eventos estressores mais frequentes e de maior impacto para os adolescentes, a partir de uma lista de 20 eventos. Os eventos estressores mais frequentes foram: alguém importante ter falecido $(77,9 \%)$; ter sido levado para o Conselho Tutelar (70,9\%); pai ou mãe teve filhos com outros parceiros $(58,4 \%)$; alguém da família estar desempregado (48,7\%). As situações apontadas pelos adolescentes acolhidos como mais estressantes (de maior impacto) foram: alguém importante ter falecido $(\mathrm{n}=84 ; \mathrm{M}=4,63 ; \mathrm{dp}=0,86)$; ter sofrido algum acidente grave $(n=21 ; M=4,33 ; d p=0,91)$; ter passado fome $(n=36 ; M=4,08 ; d p=1,18)$; ter ficado em instituição de privação de liberdade $(n=4 ; M=4 ; d p=0,82)$. Quanto a estar vivendo em instituição de acolhimento, 40,7\% considerou tal evento como "nada ruim" e $20,9 \%$ como "horrível".

A Tabela 2 apresenta a frequência e percentual de adolescentes que vivenciaram situações de violência intrafamiliar e extrafamiliar por sexo. Pode-se observar que quase $50 \%$ dos participantes já vivenciou situações de violência física, envolvendo soco ou surra, ou ainda agressão com objetos, sendo que 16,2\% dos participantes sofreram ao menos um dos tipos mencionados. Foram observadas frequências mais altas entre as meninas, na ocorrência de todos os tipos de violência intrafamiliar, com associação significativa. Os principais perpetradores foram os padrastos, para a violência sexual e os pais e irmãos para as demais agressões. Situações de violência extrafamiliar ocorreram ao menos em uma modalidade para $33,6 \%$ dos participantes. As vivências de agressão física e sexual extrafamiliar têm como principal perpetrador pessoas desconhecidas e as de ameaça ou humilhação tiveram como protagonistas, sobretudo os colegas de escola. Já nas experiências de soco ou surra os principais responsáveis foram os próprios amigos, além de colegas. No âmbito extrafamiliar não houve associação entre o sexo e qualquer tipo de violência.

Tabela 2

Frequências e Percentuais de Violência Intra e Extrafamiliar

\begin{tabular}{|c|c|c|c|c|c|c|c|c|}
\hline \multirow[t]{3}{*}{ Violência } & \multicolumn{8}{|c|}{$f(\%)$} \\
\hline & \multicolumn{4}{|c|}{ Intrafamiliar } & \multicolumn{4}{|c|}{ Extrafamiliar } \\
\hline & M & $F$ & Total & $x^{2}$ & M & $F$ & Total & $x^{2}$ \\
\hline Ameaça ou humilhação & $\begin{array}{c}9 \\
(20,9)\end{array}$ & $\begin{array}{c}29 \\
(44,6)\end{array}$ & $\begin{array}{c}38 \\
(35,2)\end{array}$ & $6,37^{*}$ & $\begin{array}{c}17 \\
(39,5)\end{array}$ & $\begin{array}{c}32 \\
(48,5)\end{array}$ & $\begin{array}{c}49 \\
(45,0)\end{array}$ & 0,84 \\
\hline Soco ou surra & $\begin{array}{c}17 \\
(39,5)\end{array}$ & $\begin{array}{c}39 \\
(60,0)\end{array}$ & $\begin{array}{c}56 \\
(51,9)\end{array}$ & $4,34^{* *}$ & $\begin{array}{c}18 \\
(42,9)\end{array}$ & $\begin{array}{c}22 \\
(32,8)\end{array}$ & $\begin{array}{c}40 \\
(36,7)\end{array}$ & 1,12 \\
\hline Agressão com objeto & $\begin{array}{c}15 \\
(34,9)\end{array}$ & $\begin{array}{c}40 \\
(61,5)\end{array}$ & $\begin{array}{c}55 \\
(50,9)\end{array}$ & $7,35^{\star}$ & $\begin{array}{c}9 \\
(20,9)\end{array}$ & $\begin{array}{c}14 \\
(20,9)\end{array}$ & $\begin{array}{c}23 \\
(20,9)\end{array}$ & 0,00 \\
\hline $\begin{array}{l}\text { Toque no corpo } \\
\text { Sem permissão }\end{array}$ & $\begin{array}{c}4 \\
(9,5)\end{array}$ & $\begin{array}{c}20 \\
(31,3)\end{array}$ & $\begin{array}{c}24 \\
(22,6)\end{array}$ & $6,83^{*}$ & $\begin{array}{c}2 \\
(4,7)\end{array}$ & $\begin{array}{c}9 \\
(13,4)\end{array}$ & $\begin{array}{c}11 \\
(10,0)\end{array}$ & 2,25 \\
\hline Relação sexual forçada & $\begin{array}{c}4 \\
(9,5)\end{array}$ & $\begin{array}{c}13 \\
(20,6)\end{array}$ & $\begin{array}{c}17 \\
(16,2)\end{array}$ & 2,29 & $\begin{array}{c}1 \\
(2,3)\end{array}$ & $\begin{array}{c}7 \\
(10,6)\end{array}$ & $\begin{array}{c}8 \\
(7,3)\end{array}$ & - \\
\hline
\end{tabular}

Notas: M: Sexo masculino; F: Sexo feminino; ${ }^{*} p<0,01 ;{ }^{* *} p<0,04$.

\section{Comportamentos de risco: relação sexual sem proteção, suicídio e uso de drogas}

Entre os participantes, $46,3 \%$ já iniciaram vida sexual ativa. A primeira relação sexual ocorreu entre 12 a 14 anos para $67,4 \%$ desses adolescentes, sendo que a faixa etária dessa iniciação variou de quatro a 17 anos. O intercurso sexual foi forçado para $24 \%$ das que já têm uma vida sexual, sendo que todas que relataram esse fato eram meninas $(n=12)$. Quanto ao uso de preservativo, no último ano, apenas $51,2 \%$ dos adolescentes sexualmente ativos fez uso de camisinha em todas as relações sexuais. A maioria daqueles adolescentes que só usa de vez em quando ou que nunca usa preservativo justificou tal prática alegando que não tinha o preservativo no momento do ato sexual $(47,4 \%)$. Em relação à ideação suicida 37 (33,9\%) participantes referiram já ter pensado em se matar, sendo observada diferença significativa por sexo $\left(\chi^{2}=10,93 ; p<0,001\right)$, com um maior percentual entre as meninas. Na mesma direção, constatou-se uma associação significativa $\left(\chi^{2}=4,89 ; p<0,03\right)$ entre ser 
menina e tentativa de suicídio. Dos 22 participantes que afirmaram já terem tentado tirar sua vida, 18 $(81,8 \%)$ eram meninas, o que corresponde a $26,47 \%$ do total das meninas.

O uso regular de substâncias psicoativas, por sua vez, foi admitido por $48,7 \%$ dos adolescentes que disseram ter consumido bebida alcoólica e por $40 \%$ que utilizaram cigarro (tabaco) no último ano. Já entre as drogas ilícitas, mais de $15 \%$ dos adolescentes aco- lhidos usava alguma substância psicoativa regularmente, como se pode observar na Tabela 3 , sendo que a idade de primeira experimentação para algumas dessas foi de quatro anos (tabaco e cola). Não foram observadas diferenças por sexo no consumo de drogas. Um teste qui-quadrado mostrou associação entre ter iniciado a vida sexual e uso de álcool $\left(\chi^{2}=4,89\right.$; $\mathrm{p}<0,03)$ e tabaco $\left(\chi^{2}=15,27 ; \mathrm{p}<0,01\right)$.

Tabela 3

Frequência e Percentual de Uso de Substâncias Psicoativas no Último Ano e Média de Idade de Experimentação

\begin{tabular}{lcl}
\hline Droga & $\boldsymbol{f ( \% )}$ & Idade de experimentação \\
\hline Álcool & $37(48,7 \%)$ & $\mathrm{M}=12,38 ; \mathrm{dp}=2,85$ \\
Tabaco & $30(40 \%)$ & $\mathrm{M}=11,98 ; \mathrm{dp}=3,03$ \\
Maconha & $12(17,4 \%)$ & $\mathrm{M}=13,24 ; \mathrm{dp}=2,28$ \\
Cocaína & $9(12,9 \%)$ & $\mathrm{M}=13,45 ; \mathrm{dp}=1,70$ \\
Cola/ solventes & $3(4,2 \%)$ & $\mathrm{M}=10,38 ; \mathrm{dp}=3,58$ \\
Crack & $2(2,9 \%)$ & $\mathrm{M}=12,50 ; \mathrm{dp}=1,73$ \\
\hline
\end{tabular}

\section{DISCUSSÃO}

A partir dos dados coletados pode-se perceber que o tempo de institucionalização observado entre os participantes deste estudo é superior ao recomendado pela Lei 12.010 (2009), o que é entendido como um indicador de fator de risco para o desenvolvimento (Carvalho, 2002). O tempo extenso de acolhimento pode enfraquecer ainda mais o vínculo familiar e até inviabilizar a reinserção à família de origem, que é o destino de muitos adolescentes acolhidos que completam 18 anos de idade (Brasil, 1990; Silva, 2004). Os potenciais efeitos nocivos do prolongado tempo de acolhimento institucional têm sido amplamente considerados na literatura especializada (Bronfenbrenner, 1979/1996; Oliveira, \& Milnitky-Sapiro, 2007; Sigal e cols., 2003; Siqueira, \& Dell'Aglio, 2006; Siqueira, \& Dell'Aglio, 2010; Siqueira, Zoltowski, Giordani, Otero, \& Dell'Aglio, 2010). Entretanto, percebe-se que os dados do presente estudo estão mais promissores em relação ao último registro em nível nacional (Levantamento Nacional de Abrigos Brasileiros para Crianças e Adolescentes), que indicou a variação de um período de 10 anos na institucionalização de crianças e adolescentes (Silva, 2004). Resultado semelhante foi observado por Cavalcante, Magalhães e Pontes (2007) e Siqueira e Dell’Aglio (2010).
A relação entre a idade maior que tinha o adolescente ao ser acolhido pela primeira vez com a maior frequência de contato com família de origem durante a sua permanência na instituição é um dado que confirma a visão de diversos autores sobre o enfraquecimento dos vínculos à medida que passam os anos de institucionalização, sobretudo quando a idade de entrada na instituição é mais precoce (Carvalho \& Manita, 2010; Paião, 2010). Dessa forma, é possível que a qualidade das relações com a família fique mais prejudicada quando a criança é acolhida mais precocemente, uma vez que há menos tempo para que se estabeleçam processos proximais nesse contexto (Bronfenbrenner, 2011). De fato, as crianças que nunca experimentaram alguma forma de vínculo são as que mais prejuízos sofrem no desenvolvimento (Howe, 1995). Independentemente da idade de entrada na instituição, Silva (2012) relembra que o tempo no interior de uma instituição possui uma percepção subjetiva além de cronológica. Assim, a discussão sobre o tempo necessário de acolhimento ou o momento de retorno às famílias deve ser pensada em conjunto com o adolescente, sua família, os técnicos e demais profissionais envolvidos.

Destaca-se ainda a questão da convivência com irmãos, já que os dados indicam que 45,1\% dos participantes possui irmãos na mesma instituição. Esta 
configuração cumpre com as orientações do ECA (Brasil, 1990), amparado também pela Lei 12.010/09 (Brasil, 2009), que preconizam a não separação de irmãos no momento da institucionalização, buscando manter e reforçar o vínculo familiar de crianças e adolescentes. Considerando a pouca chance dos adolescentes de virem a ser adotados caso as famílias percam o poder legal sobre os mesmos, investir na convivência entre irmãos não abusivos pode significar um resgate de sua história e identidade que são essenciais para o pleno desenvolvimento dos processos proximais (Bronfenbrenner, 2011).

Com relação ao perfil sociodemográfico, constatou-se que a predominância de adolescentes negros e pardos e de origem sociofamiliar vulnerável repete os percentuais de diversos levantamentos e estudos (Gasperin e cols., 2007; Paião, 2010; Silva, 2004), inclusive em relação ao grande número de irmãos também acolhidos. Esse dado revela a importância do aspecto social e político da assistência às famílias vulneráveis e também do ainda pobre alcance das intervenções políticas e legais. Vale lembrar que o ECA aponta que a pobreza, por si só, não deve ser motivo de medidas de proteção como o acolhimento institucional (Brasil, 1990). Quanto à predominância do sexo feminino entre as adolescentes acolhidas participantes do estudo, trata-se de uma informação contrária aos estudos anteriores (Gasperin e cols., 2007; Paião, 2010; Silva, 2004), em que os meninos são a maioria. Entretanto, cabe ressalvar que os levantamentos existentes sobre o perfil de acolhidos em geral não diferenciam as faixas etárias, apresentando percentuais reunidos de crianças e adolescentes, o que poderia explicar tais discrepâncias a respeito do sexo. Uma ligeira predominância do sexo feminino ocorreu na faixa etária acima de 16 anos no estudo de Coelho, Hirata, Barbette, Vieira e Oliveira (2009), com acolhidos da região metropolitana de Campinas (SP).

O último levantamento realizado no Brasil em 2003 revelou que $87 \%$ das crianças e adolescentes acolhidos tinham família, sendo que 58,2\% mantinham vínculos familiares (Silva, 2004). Dados regionais de 2007 indicaram que menos de $20 \%$ das crianças e adolescentes recebiam visita na região metropolitana de Porto Alegre. A Lei 12.010 (Brasil, 2009) preconiza que crianças e adolescentes tenham garantida a convivência com suas famílias de origem durante o período de acolhimento institucional e que esse tempo não ultrapasse dois anos. Considerando o ex- pressivo percentual de adolescentes que mantêm contato com a família, sobretudo recebendo visitas (cerca de $70 \%$ ), pode-se pensar que esse aspecto do contexto institucional pode ter melhorado em relação aos dados divulgados em 2007 (Gasperin e cols., 2007), ainda que o tempo de acolhimento continue extrapolando a transitoriedade prevista no ECA.

A escola, que poderia representar para alguns dos adolescentes um espaço potencial para formação de vínculos, mostrou-se um tanto falha em seu papel protetivo na medida em que revela altos percentuais de reprovação e expulsão. Além disso, a escola foi referida como um lugar onde estão expostos à ameaça e humilhação. Os fatores de estresse envolvidos no rompimento total ou parcial dos vínculos familiares normalmente estão relatados na literatura como contribuindo para o desenvolvimento de dificuldades escolares e esse problema pode estar relacionado ao alto índice de reprovação entre os participantes (Middelton-Moz \& Zawadski, 2007; Stevanato, Loureiro, Linhares, \& Marturano, 2003). Além disso, ser vítima ou testemunha de violência também está relacionado a problemas cognitivos (Sigal, Perry, Rossignol, \& Ouimet, 2003; Velarde \& Martínez, 2008).

No contexto intrafamiliar, os percentuais observados de exposição à violência são preocupantes, principalmente se comparados a percentuais entre adolescentes que vivem em outros contextos (Braga, 2011; Wathier \& Dell'Aglio, 2007). Esse dado demonstra, contudo, que as determinações do ECA (Brasil, 1990) estão sendo cumpridas, tendo em vista que esses adolescentes se encontram em medida protetiva. Com relação ao abuso sexual no contexto familiar, vivenciado pelas meninas do presente estudo, confirmam-se os perfis de famílias abusivas (Pelisoli, Pires, Almeida \& Dell'Aglio, 2010; Sanderson, 2005) em que mais de $70 \%$ dos casos envolveram o sexo feminino como vítima e os principais abusadores foram os padrastos $(19,6 \%)$ e pais $(17,1 \%)$. Destaca-se que é importante não culpabilizar as características da família como exclusivamente determinantes da violência, como alertaram Santos, Pelisoli e Dell'Aglio (2012) e também Sanderson (2005), segundo a qual, é preciso preparar as escolas e outras instituições que trabalham com crianças e adolescentes para que consigam protegê-los da violência sexual. Outro aspecto importante sobre a questão do sexo é a ausência de afirmações sobre abuso sexual entre os meninos participantes. Uma vez que o presente estudo valeu-se de autores- 
postas, os meninos podem ter tido mais empecilhos para revelar esta violência devido ao preconceito e questões culturais, confirmando o exposto na literatura (Hohendorff, Bavaresco, Habigzang \& Koller, 2012; Sanderson, 2005), pois os percentuais epidemiológicos chegam a estimar que um em cada seis meninos experimentou alguma forma de abuso sexual (Sanderson, 2005), enquanto a proporção para meninas seria de um para quatro.

Quanto ao uso de drogas, os percentuais de uso de álcool e tabaco identificado no último ano confirmam diversos estudos que apontam o uso dessas drogas entre adolescentes (Galduroz, Noto, Fonseca, \& Carlini, 2005; Laranjeira, Pinsky, Zaleski, \& Caetano, 2007; Vieira, Ribeiro \& Laranjeira, 2007). Em estudo com adolescentes brasileiros foi observado um percentual de 49,8\% de uso de álcool e $20,1 \%$ de uso de tabaco (Carlini, Galduróz, Noto, \& Nappo, 2002). Neste estudo foi observada associação entre o consumo de álcool e tabaco no último ano e a vida sexual ativa dos adolescentes. Para Ferreira e Torgal (2011), que também observaram essa associação, o uso de álcool e tabaco pode facilitar o envolvimento de adolescentes em relações sexuais, sobretudo sem preservativos, o que os expõe potencialmente ao risco de contaminação por doenças sexualmente transmissíveis (Ferreira \& Torgal, 2011). Comparados ao levantamento entre adolescentes em todo o país $(48,3 \%$ e $15,7 \%$ ), o uso contínuo de álcool e tabaco pelos participantes deste estudo foi superior $(48,7 \%$ e $40 \%$, respectivamente). Quanto às drogas ilícitas, os percentuais também foram mais altos do que de adolescentes brasileiros em 2001. Por exemplo, no levantamento brasileiro o uso da maconha ficou em $3,5 \%$, dos solventes, 3,4\% e da cocaína, $0,5 \%$ (Carlini et al., 2002), ao passo que, neste estudo os percentuais foram, respectivamente, $17,4 \%, 4,2 \%$ e $12,9 \%$.

Os dados sobre comportamentos de risco, como prática sexual insegura, elevado percentual de tentativas de suicídio e uso de drogas podem estar refletindo os efeitos da exposição à violência que, na maioria das vezes, é o motivo do acolhimento institucional, assim como a fragilidade emocional presente entre os adolescentes em acolhimento.

\section{CONCLUSÃO}

Esses dados permitem concluir que estes adolescentes estão expostos a um grande número de fatores de risco ao desenvolvimento e que apresentam fragilidade nos vínculos com suas famílias. Há uma discrepância entre a exigência legal de que a instituição promova a convivência familiar sempre que possível e a disfuncionalidade familiar ou a precariedade social que contribuiu para o desgaste destas relações, motivando o acolhimento. Ainda que exista legislação com orientações sobre os aspectos mínimos a serem cumpridos para que a instituição de acolhimento seja de fato um contexto que favoreça a continuidade do desenvolvimento integral, da mesma forma que acontece com outras leis brasileiras, ainda há necessidade de novas regulamentações, assim como a efetiva aplicação do que está previsto nestas leis.

O presente estudo contribui principalmente na identificação de fatores de risco presentes no desenvolvimento de adolescentes acolhidos, de forma que possam ser propostas intervenções junto a essa população. Contudo, para estudos futuros, sugere-se a utilização de coletas de dados junto aos técnicos da instituição ou prontuários dos adolescentes para que seja possível uma triangulação dos dados que compõem o perfil do acolhido, garantindo maior validade ecológica aos dados. Além disso, são necessários estudos longitudinais, que possam avaliar mais sistematicamente o impacto da institucionalização sobre o desenvolvimento desses jovens.

A vulnerabilidade observada entre os participantes deste estudo, relacionada a múltiplas exposições a fatores de risco ao desenvolvimento, revela o grande desafio que se impõe às instituições de acolhimento, bem como para os profissionais que ali trabalham e respectivos gestores. Dessa forma, torna-se necessária uma capacitação permanente para os profissionais que atuam junto a esta população, tendo em vista suas especificidades. Além disso, também é evidenciada a necessidade de políticas públicas e de investimento em intervenções que auxiliem na recuperação e fortalecimento dos laços familiares, bem como em ações preventivas à institucionalização.

\section{REFERÊNCIAS}

Albornoz, A. C. G. (2009). Perspectivas no abrigamento de crianças e adolescentes vitimizados. Em S. L. R. Rovinski \& R. M. Cruz (Orgs.), Psicologia jurídica: Perspectivas teóricas e processos de intervenção (pp.181-194). São Paulo: Vetor.

Alexandre, D. T., \& Vieira, M. L. (2004). Relação de apego entre crianças institucionalizadas que vivem em situação de abrigo. Psicologia em Estudo, Maringá, 9(2), 207-217. 
Arpini, D. M., \& Lopes, M. D. C. (2009). Retratos da infância: um olhar voltado às instituições de abrigo. Em D. M. Arpini (Org.), Psicologia, família e instituição (pp.89-128). Santa Maria: Editora UFSM.

Azor, A. M. G., \& Vectore, C. (2008). Abrigar/desabrigar: Conhecendo o papel das famílias nesse processo. Estudos de Psicologia (Campinas), 25(1), 77-89.

Braga, L. de L. (2011). Exposição à violência e comportamento suicida em adolescentes de diferentes contextos. Dissertação de Mestrado não publicada. Programa de Pós-Graduação em Psicologia, Universidade Federal do Rio Grande do Sul. Porto Alegre, RS.

Brasil (1990). Estatuto da Criança e do Adolescente. Diário Oficial da União. Lei $n^{o}$ 8069, de 13 de julho de 1990. Brasília, DF.

Brasil, Casa Civil. (2009). Lei $n^{o} 12.010$, de 3 de agosto, Retirado de http://www.planalto.gov.br/ccivil_03/_Ato2007-2010/2009/ Lei/L12010.htm em 10/04/2011

Bronfenbrenner, U. (1996). A ecologia do desenvolvimento humano. Porto Alegre: Artes Médicas. Original publicado em 1979.

Bronfenbrenner, U. (2011). Bioecologia do desenvolvimento humano: tornando os seres humanos mais humanos. Porto Alegre: Artmed. Original publicado em 2005.

Carlini, E. A., Galduróz, J. C. F., Noto, A. R., \& Nappo, S. A. (2002). I Levantamento domiciliar sobre o uso de drogas psicotrópicas no Brasil: estudo envolvendo as 107 maiores cidades do país: 2001/São Paulo: CEBRID/UNIFESP.

Carvalho, A. M. (2002). Crianças institucionalizadas e desenvolvimento: Possibilidades e desafios. Em E. R. Lordelo, A. M. A. Carvalho, \& S. H. Koller (Orgs.), Infância brasileira e contextos de desenvolvimento (pp. 19-44). São Paulo: Casa do Psicólogo: Salvador, BA: Editora da Universidade Federal da Bahia.

Carvalho, T., \& Manita, C. (2010). Percepções de crianças e adolescentes institucionalizados sobre o processo de institucionalização e a experiência na instituição. Em C. Nogueira, I. Silva, L. Lima, A. T. Almeida, R. Cabecinhas, R. Gomes, C. Machado, A. Maia, A. Sampaio \& M. C. Taveira (Orgs.), Actas do VII Simpósio Nacional de Investigação em Psicologia (pp. 33263335), Retirado de http://www.actassnip2010.com em $10 / 04 / 2011$

Cavalcante, L. I. C., Magalhães, C. M. C., \& Pontes, F. A. R. (2007). Institucionalização precoce e prolongada de crianças: discutindo aspectos decisivos para o desenvolvimento. Alethéia, 25, 20-34.

Coelho, R. P. de S., Hirata, R. M., Barbette, M. H., Vieira, M. O., \& Oliveira, M. de. (2009). Perfil das crianças e adolescentes em acolhimento institucional na região metropolitana de campinas: Texto para discussão do observatório de convivência familiar e comunitária. São Paulo: Universidade Estadual de Campinas, Núcleo de Estudos de Políticas Públicas.

Dalbem, J. X., \& Dell'Aglio, D. D. (2008). Apego em adolescentes institucionalizadas: processos de resiliência na formação de novos vínculos afetivos. Psico, 39(1), 33-40.

Ferreira, M. M. da S. R. dos S., \& Torgal, M. C. L. de F. P. R. (2011) Estilos de vida na adolescência: Comportamento sexual dos adolescentes portugueses. Revista da Escola de Enfermagem da USP, 45(3), 589-95.

Fundação de Proteção Especial do Rio Grande do Sul (FPERGS). (2012, maio). Apresentação FPERGS. Relatório apresentado no Seminário FPERGS: 10 anos trabalhando para a garantia dos direitos das crianças e adolescentes do RS. Sala João Neves da Fontoura, Plenarinho da Assembleia Legislativa. Porto Alegre, RS.

Galduroz, J. C. F., Noto, A. R., Fonseca, A. M., \& Carlini, E. A. (2005). V levantamento nacional sobre o consumo de drogas psicotrópicas entre estudantes do ensino fundamental e médio da rede pública de ensino nas 27 capitais brasileiras: 2004. São Paulo: CEBRID/ UNIFESP.

Gasperin, C., Luz, C. M. da, Souza, F. R. S. de, Perin, S. D., \&Tejadas, S. da S. (2007). Rede de abrigos de Porto Alegre: um olhar sobre a realidade. In Rio Grande do Sul. Ministério Público (Org.), Rede de abrigos de Porto Alegre: um olhar sobre a realidade (pp. 24-115). Porto Alegre: Procuradoria-Geral de Justiça.

Guará, I. M. F. (2006). Abrigo: Comunidade de acolhida e socioeducação. Em M. V. Baptista (Org.), Abrigo: Comunidade de acolhida e socioeducação (pp. 63-75). São Paulo: Instituto Camargo Corrêa.

Hohendorff, J. V., Bavaresco, P. D., Habigzang, L. F., \& Koller, S. H. (2012). Abuso sexual contra meninos: Uma revisão. Em L. F. Habigzang, \& S. H. Koller (Orgs.), Violência contra crianças e adolescentes: Teoria, pesquisa e prática (pp.107-122). Porto Alegre: Artmed.

Howe, D. (1995). Attachment theory for social work practice. London: Macmillan Press.

Laranjeira, R., Pinsky, I., Zaleski, M., \& Caetano, R. (2007). I Levantamento nacional sobre os padrões de consumo de álcool na população brasileira. Brasília: Secretaria Nacional Antidrogas.

Middelton-Moz, J. \& Zawadski, M. L. (2007). Bullying: Estratégias de sobrevivência para crianças e adultos. Porto Alegre: Artmed.

Oliveira, A. P., \& Milnitky-Sapiro, C. (2007). Políticas públicas para adolescentes em vulnerabilidade social: abrigo e provisoriedade. Psicologia: Ciência e Profissão, 27, 622-635.

Oliveira, S. V. \& Próchno, C. C. S. C. (2010). A vinculação afetiva para crianças institucionalizadas à espera de adoção. Psicologia: Ciência e Profissão, 30(1), 62-84.

Paião, I. C. F. (2010). O desacolhimento institucional de crianças e adolescentes e o serviço social. Dissertação de Mestrado não publicada. Programa de Pós-Graduação em Serviço Social e Políticas Sociais, Universidade Estadual de Londrina. Londrina, PR.

Prada, C. G., \& Weber, L. N. D. (2002). O abrigamento de crianças e adolescentes: Proteção ou esquecimento? Em Associação Brasileira de Psicoterapia e Medicina Comportamental, Anais do XI Encontro da Associação Brasileira de Psicoterapia e Medicina Comportamental, Londrina, PR. 
Prada, C. G., Williams, L. C., \& Weber, L. N. D. (2007). Abrigos para crianças vítimas de violência doméstica: Funcionamento relatado pelas crianças e pelos dirigentes. Psicologia: Teoria e Prática, 9(2), 14-25.

Ribeiro, M. O., \& Ciampone, M. H. T. (2002). Crianças em situação de rua falam sobre os abrigos. Revista da Escola de Enfermagem da USP, 36(4), 309-316.

Sanderson, C. (2005). Abuso sexual em crianças: Fortalecendo pais e professores para proteger crianças contra abusos sexuais e pedofilia. São Paulo: MBooks.

Santos, S. S. dos, Pelisoli, C., \& Dell'Aglio, D. D. (2012). Desvendando segredos e dinâmicas familiares no abuso sexual infantil. Em L. F. Habigzang, \& S. H. Koller (Orgs.), Violência contra crianças e adolescentes: Teoria, pesquisa e prática (pp.55-68). Porto Alegre: Artmed.

Sigal, J., Perry, J. C., Rossignol, M. I., \& Ouimet, M. C. (2003). Unwanted infants: psychological and physical consequences of inadequate orphanage care 50 years later. American Journal of Orthopsychiatry, 73(1), 3-12.

Silva, E. R. A. (2004). O direito à convivência familiar e comunitária: Os abrigos para crianças e adolescentes no Brasil. Brasília: IPEA/ Conanda.

Silva, M. L. (2012). Lei nacional de adoção e acolhimento institucional: $O$ ponto de vista de psicólogos e assistentes sociais. Dissertação de Mestrado não publicada. Programa de PósGraduação em Psicologia, Universidade Federal de Santa Maria. Santa Maria, RS.

Siqueira, A. C. \& Dell'Aglio, D. D. (2006). O impacto da institucionalização na infância e na adolescência: Uma revisão de literatura. Psicologia \& Sociedade, 18, 71-80.
Siqueira, A. C. \& Dell'Aglio, D. D. (2010). Crianças e adolescentes institucionalizados: Desempenho escolar, satisfação de vida e rede de apoio. Psicologia: Teoria e Pesquisa, 26(3), 407-415.

Siqueira, A. C., Zoltowski, A. P., Giordani, J., Otero,T., \& Dell'Aglio, D. D. (2010). Processo de reinserção familiar: estudo de casos de adolescentes que viveram em instituição de abrigo. Estudos de Psicologia (Natal), 15, 07-15.

Stevanato, I. S., Loureiro, S. R., Linhares, M. B. M., \& Marturano, E. M. (2003). Autoconceito de crianças com dificuldades de aprendizagem e problemas de comportamento. Psicologia em Estudo, 8(1), 67-76.

Velarde, M., \& Martínez, P. (2008). Perspectiva temporal futura em adolescentes institucionalizados. Revista de Psicologia, $X X V I(2), 256-276$.

Vieira, D. L., Ribeiro, M. R., M., \& Laranjeira, R. R. (2007). Álcool e adolescentes: estudo para implementar políticas municipais. Revista de Saúde Pública, 41(3), 396-403.

Wathier, J. L., \& Dell'Aglio, D. D. (2007). Sintomas depressivos e eventos estressores em crianças e adolescentes no contexto de institucionalização. Revista de Psiquiatria do Rio Grande do Sul, 29(3), 305-314.

Weber, L. N. D. (2007). Eduque com carinho. $2^{\mathrm{a}}$ ed. Curitiba: Juruá. 